\title{
TRANSFORMATION DU POLITIQUE SOUS LE REGARD DES MÉDIAS
}

\author{
Pascal Delwit, Jean-Benoit Pilet et Nicolas De Decker ${ }^{1}$
}

Observer que l'influence des medias sur la vie politique actuelle est prépondérante est un constat qui peut sembler évident. Néanmoins, l'importance du phénomène justifie de se pencher plus en détails sur cette évolution pour tenter d'en cerner les contours et les effets. En l'occurrence, pour de nombreux auteurs, la prégnance des médias et, a fortiori de la télévision, est une des caractéristiques majeures de la politique depuis les années $1960^{2}$. Selon Pippa Norris, la centralité des médias dans le jeu politique est même la spécificité, le cœur de la politique moderne. Certains auteurs postulent même une intrication congénitale de la politique et de la communication, donc des médias. Ainsi pour Breton, comme pour d'autres sociologues des médias, la politique à l'ère démocratique est intrinsèquement communication. Sinon, elle n'est pas. Reste que l'irruption de la télévision dans les foyers bouleverse incontestablement la donne médiatique, et, partant, politique, dès

1 Centre d'étude de la vie politique (Cevipol), Université libre de Bruxelles.

2 Keane, John, The Media and Democracy, Blackwell, Oxford, 1991. 
la fin des années 1960, période à partir de laquelle le petit écran entame sa domination du système médiatique, reléguant presse et radio, autrefois dominantes, à la remorque de l'audiovisuel ${ }^{1}$.

Nombreux sont les spécialistes à adopter une vision trilogique de l'histoire des médias. Thoveron en décrit ainsi trois âges successifs, traversant respectivement les dix-neuvième, vingtième, et vingt-etunième siècle : l'âge de la presse d'opinion, l'âge de la presse d'information, et l'âge du spectacle ${ }^{2}$. Reprenant la même structure trilogique, et la corrélant aux pratiques politiques, une politiste comme Pippa Norris distingue trois ères : pré-moderne, moderne et post-moderne (figure 1) ${ }^{3}$. Au cours de la période pré-moderne, la politique se caractérise par une communication et des campagnes gérées au niveau local par les partis et les militants. Ceux-ci sont la source principale d'information politique au travers des tracts, des meetings et des journaux partisans.

Figure 1 : les trois ères de la politique

\begin{tabular}{|l|l|l|l|}
\hline & Pré-moderne & Moderne & Post-moderne \\
\hline $\begin{array}{l}\text { Gestion de la } \\
\text { campagne }\end{array}$ & décentralisée & centralisée & décentralisée \\
\hline $\begin{array}{l}\text { Canaux de } \\
\text { communication }\end{array}$ & $\begin{array}{l}\text { Presse partisane } \\
\text { Meetings } \\
\text { Tracts }\end{array}$ & $\begin{array}{l}\text { Télévision } \\
\text { Presse indépen- } \\
\text { dante } \\
\text { Débats télévisés }\end{array}$ & $\begin{array}{l}\text { Télévision } \\
\text { Sites web } \\
\text { E-mails } \\
\text { Forums on-line }\end{array}$ \\
\hline
\end{tabular}

À cette ère succède la période moderne dont le trait distinctif est que la vie politique se structure autour de médias indépendants, de la presse bien sûr, mais aussi et surtout de la télévision. Les campagnes deviennent nationales et se mènent dans des médias pluralistes qui supplantent la presse partisane. Enfin, Norris voit émerger les prémices d'une ère post-moderne où les nouvelles technologies de l'information supplanteront les médias classiques et permettront de décentraliser les campagnes pour adapter le message à chaque électeur.

1 Guillebaud, Jean-Claude, «La question médiatique », in: Le Débat, $\mathrm{n}^{\circ}$ 138, janvierfévrier 2006, p. 103.

2 Thoveron, Gabriel, Le troisième âge du quatrième pouvoir, Bruxelles, Labor, 1999.

3 Norris, Pippa, «Campaign Communication », in LeDuc, Lawrence, Niemi, Richard G. and Norris, Pippa (eds.), Comparing Democracies 2, Sage, London, 2002, pp. 127-147. 
Partageant ce constat d'une centralité des médias, et en particulier de la télévision, dans la vie politique contemporaine, de nombreux chercheurs en science politique ont développé des recherches sur les rapports entre médias et politique. Ces études ont principalement suivi deux directions. D'un côté, de nombreux ouvrages ont été consacrés aux fonctions des médias dans le système politique. De l'autre, plusieurs analyses ont étudié les effets de la médiatisation sur la vie politique dans les démocraties. Afin de planter le décor de cette étude, les principaux enseignements des deux approches des rapports entre médias et politiques vont être présentés.

En ce qui concerne les fonctions des médias dans la vie démocratique, celles-ci sont de trois ordres. La première, la plus évidente, est l'information. La presse et la télévision ont pour mission de transmettre aux citoyens ce qui est proposé, décidé et mis en œuvre par les responsables politiques. À cet égard, il apparaît que la télévision se taille la part de lion pour ce qui a trait à la fonction d'information. Ainsi, comme le note Negrine, la télévision est de loin le média de référence pour les informations politiques en Grande-Bretagne ${ }^{1}$.

Tableau 1 : Sources d'information politique

\begin{tabular}{|l|c|c|}
\hline & $\begin{array}{l}\text { Source d'information } \boldsymbol{n}^{\circ} \mathbf{1} \\
(\%)\end{array}$ & $\begin{array}{l}\text { Parmi les 2 premières } \\
\text { sources d'information (\%) }\end{array}$ \\
\hline $\boldsymbol{T V}$ & 63 & 88 \\
\hline Presse écrite & 29 & 73 \\
\hline Radio & 4 & 14 \\
\hline
\end{tabular}

Comme le démontre le tableau 1 , près de deux tiers des citoyens britanniques citent la télévision comme première source d'information politique. La presse écrite arrive en seconde position avec uniquement $29 \%$. Les quotidiens semblent être surtout une source d'information complémentaire sachant que $44 \%$ des personnes interrogées classent la presse écrite comme seconde source d'information. La radio vient dans tous les cas loin derrière avec $4 \%$ des Britanniques qui la citent comme première source d'information. Le constat vaut pour l'ensemble des pays industrialisés ${ }^{2}$. Le grand paradoxe de cette primauté de la télévi-

\footnotetext{
Negrine, Ralph, Politics and the Mass Media in Britain

Delporte, Christian, « De la propagande à la communication », in: Le Débat, n 138 ,
} 
sion comme moyen d'information vient du discrédit massif qui accable la folle du logis quant à sa faculté de proposer une information fiable. Les enquêtes sur la confiance du public envers les médias, telles celle que publie annuellement Télérama, situent en effet systématiquement la télévision loin derrière presse écrite et radio dès lors que la variable informationnelle est prise en considération...

La deuxième fonction des médias est d'accueillir, de relayer et de stimuler le débat public. En ce sens, la presse et en particulier la télévision remplacent le Parlement comme lieu privilégié de la confrontation entre adversaires politiques. Comme l'a notamment démontré Iyengar, les citoyens se représentent le jeu politique en fonction du reflet qu'ils en ont lors des débats télévisés ${ }^{1}$. Le cas des élections présidentielles en est la meilleure illustration. Le moment clef d'une campagne présidentielle est le débat entre les deux concurrents retransmis à la télévision. Plus que tout meeting électoral ou tout débat parlementaire, la controverse retransmise sur les écrans TV va construire l'image que les citoyens ont de la compétition pour l'accession à la présidence. "Le développement et surtout la diffusion des nouveaux moyens modernes de communication, qui deviennent des points de passage obligés de la carrière des hommes politiques ayant une ambition nationale, ont entraîné un déplacement progressif du centre de gravité de l'espace politique, des assemblées parlementaires vers les médias ${ }^{2} »$.

Enfin, la dernière fonction, celle qui suscite d'ailleurs le plus de débat, est que les médias sont des faiseurs d'opinion. Ils ne se contentent pas de transmettre l'information politique; ils la construisent également. Comme l'expriment bien Butler et Ranney : " the role of the media has moved increasingly from being merely a channel of communication to being a major actor in the campaign process, as it seeks the persons and the issues to be covered ${ }^{3} »$.

Les exemples du rôle actif des médias dans la construction de la réalité politique sont multiples. En 1992, la campagne virulente en Grande-Bretagne des tabloïds conservateurs à l'encontre du parti travailliste a contribué à créer l'image d'un Labour radicalement de

\footnotetext{
janvier-février 2006, p. 41

1 Iyengar, Shanto, Is Anyone Responsible? How Television Frames Political Issues, University of Chicago Press, Chicago, 1991.

2 Champagne, Patrick, Faire l'opinion, Paris, Editions de Minuit, 1990, p.147.

3 Butler, D. and Ranney, A., Electioneering: A Comparative Study of Continuity and Change, Clarendon Press, Oxford, 1992, p. 283.
} 
gauche (looney left $)^{1}$. Cette campagne a alors posé la question de l'influence des médias sachant que la quasi-totalité des tabloïds populaires étaient favorables au parti conservateur ${ }^{2}$. Dans la même logique, comme l'explique Page, le soutien du Times et du Washington Post à la première guerre du Golfe a conditionné la perception de ce conflit par les citoyens américains ${ }^{3}$. Le soutien de Fox News au dernier conflit en Irak a joué dans le même sens. En Italie, la carrière politique de Silvio Berlusconi illustre à merveille le propos, tant il a prouvé, via les réseaux télévisés stipendiés (les chaînes de son groupe Mediaset), puis affidés (les chaînes publiques de la $R A I$ ) qui ont promu ses évolutions politiques, que conquérir puis exercer le pouvoir peuvent se trouver facilités dès lors que l'on contrôle, politiquement et/ou économiquement, des stations rassemblant les neuf dixièmes de l'audience de la nation que l'on tend à gouverner. Les prochaines législatives transalpines nous offrent d'ailleurs une intéressante lutte inter-médiatique, puisque, à travers le soutien plus ou moins appuyé à l'un ou l'autre des candidats en lice s'opposent, dans une homogénéité quasi parfaite, les deux grands moyens de communication du siècle dernier. La presse écrite, jusqu'au libéral Corriere della Sera ${ }^{4}$ se range en effet derrière Romano Prodi, alors que les chaînes de télévision, nul besoin de le préciser, appuyent plus ou moins discrètement Berlusconi. Au reste, entre deux candidats que tout oppose, sur la forme comme sur le fond, rien d'étonnant à ce que les messages du Professore et celui de Sua Emittenza s'expriment à travers des canaux différents.

Ce chapelet d'exemples de soutien médiatique à une cause qui triomphe ne doit pas faire inférer une influence mécanique des médias sur l'opinion, tant s'en faut. De fait, l'on pourrait multiplier à l'envi les contre-exemples d'une quasi-unanimité médiatique contredite par l'électeur, depuis Roosevelt luttant contre une presse républicaine dans sa grande majorité jusqu'au récent référendum constitutionnel français. Ce rôle actif des médias dans la construction de la réalité politique, s'il est indéniable, doit donc être tempéré à l'aune de ses traductions

1 Negrine, Ralph., op. cit.

2 Kavanagh, Dennis, Britsh Politics : Continuities and Changes, Oxford University Press, Oxford, 2000, p. 204.

3 Page, Benjamin I., Who Deliberates? Mass Media in Modern Democracy, University of Chicago Press, Chicago, 1996.

4 À l'exception des quelques titres que possède Berlusconi dans la presse quotidienne (Il Giornale surtout), mais qui ne pèsent qu'un maigre poids dans son empire médiatique. 
électorales présumées. On se trouverait même, en ce domaine, face à ce que Jacques Le Bohec qualifie de mythe journalistique. «En ce qui concerne l'influence électorale, on observe que tous les démentis offerts par les résultats électoraux et les travaux des chercheurs n'ont pas mis fin à la croyance (particulièrement bien répandue parmi les hommes politiques) dans l'importance des médias comme moyen de persuader l'opinion publique ${ }^{1} »$.

Etant conscient de la forte influence des médias sur la construction de la réalité politique, la question qui se pose pour la science politique, mais plus largement pour les sociétés démocratiques est d'établir s'il est bon de laisser l'information être transmise par des groupes médiatiques ayant des buts politiques. Une telle situation ne mène-t-elle pas à l'exclusion de certaines opinions politiques qui ne sont pas en accord avec les lignes directrices des propriétaires des grands groupes politiques ? Et, même lorsque les biais politiques ne sont pas conscients, les chaînes de télévision généralistes, par leur recherche constante du lessobjection programme ne cadenassent-elles pas l'expression politique en interdisant d'antenne les interlocuteurs les plus radicaux, ceux-là même qui sont souvent les plus novateurs ? Cette volonté motrice de rassembler le plus large auditoire, productrice d'un consensus médiatique conduit Guy Debord, avec tant d'autres, à déplorer dans une analyse déjà ancienne, cette interminable série des affrontements dérisoires ${ }^{2}$. Les nombreux contempteurs d'une pensée unique ${ }^{3}$, expression qui fit, ironie de l'histoire, florès jusque dans les cénacles médiatiques les plus convenus et dans des acceptions les plus diverses, tiennent ici leur argument le plus saillant.

Au-delà de ces débats sur les fonctions des médias dans une démocratie, la seconde ligne d'approche de la science politique par rapport aux rapports entre politique et presse concerne les effets de la médiatisation sur la vie politique. Diverses études se sont à cet égard penchées sur la manière dont le poids croissant des médias et de la télévision a fait évoluer la façon dont se fait la politique et dont ses acteurs s'organisent. "Le petit écran ne se contente pas d'offrir aux politiques qui exercent le pouvoir ou aspirent à le conquérir un contact puissant et immédiat

1 Le Bohec, Jacques, Les mythes professionnels des journalistes, Paris, L'Harmattan, 200, p. 70.

2 Debord, Guy, La société du spectacle, Paris, Gallimard, 1992.

3 Ramonet, Ignacio, «La pensée unique », in : Le Monde Diplomatique, janvier 1995, p. 1. 
avec le peuple. Il les oblige à modifier leur agenda, transformer leur langage et soigner leur apparence ${ }^{1} »$.

Pour commencer, l'omniprésence des médias et leur rôle prédominant dans la construction de la réalité politique ont modifié profondément l'action politique elle-même. En effet, afin d'exister une décision, une prise de position ou même un acteur politique doit être présent dans les médias ${ }^{2}$. Dans ces circonstances, toute action se doit d'être avant tout réfléchie par rapport à la publicité qui peut en être tirée. Une des conséquences de cette logique dictée par les médias est que les ministres et les décideurs politiques se sont de plus en plus entourés de conseillers en communication. Le poids des porte-parole s'est accru aux dépens d'autres collaborateurs.

Cette évolution s'est marquée au niveau ministériel, mais aussi au sein des partis eux-mêmes. Lorsque les médias n'étaient pas le canal dominant d'information politique, les partis devaient disposer d'une base large de militants. Ces derniers avaient pour mission d'informer les citoyens sur les actions et les prises de position de leur formation. Dans un système où les médias sont centraux, ces militants de base ont perdu leur attrait. Les partis se sont dès lors professionnalisés et ont de plus en plus engagé des conseillers en communication, des spin doctors. Le contact direct du parti avec les électeurs au travers des militants a été progressivement remplacé par des liens indirects au travers des médias et des spécialistes en relations publiques.

Le moment de la vie politique où la centralité de la communication et des médias est la plus marquante est la campagne électorale. La lutte pour l'accès aux fonctions électives s'est progressivement réformée pour répondre aux intérêts et aux besoins des médias ${ }^{3}$. En particulier, les partis ont modifié leurs techniques de campagne. De façon croissante, ils ont eu recours à des experts en communication, aux sondages d'opinion et aux spécialistes en marketing pour mener à bien une campagne électorale. Comme l'affirment Bowler et Farrell, « it is no exaggeration to state that the use of advertising companies by political parties is the norm in electoral campaigning ${ }^{4} »$. De multiples exemples viennent

1 Pingaud Denis, Poulet Bernard, « Du pouvoir des médias à l'éclatement de la scène publique », in: Le Débat, n 138, janvier-février 2006, p. 7.

2 Kavanagh, Denis, op. cit.

3 Swanson, David L. and Mancini, Paolo (eds.), Politics, Media and Modern Democracy, Praeger, London, 1996.

4 Bowler, Shaun and Farrell, David, Electoral Strategies and Political Marketing, St 
confirmer cette assertion. Les compagnies publicitaires ayant pris part aux grandes campagnes électorales dans différents pays se multiplient, avec, on le sait, plus ou moins de fortune'.

Mais plus que de modifier l'entourage des responsables politiques dans l'action politique et les campagnes électorales, les médias ont profondément bouleversé le cœur de la vie démocratique en plaçant les candidats individuels au-dessus des partis. "Ce ne sont plus les partis qui servent de relais de communication entre les décideurs politiques et les citoyens ${ }^{2} »$. Pour de nombreux auteurs, la personnalisation de la vie politique est la marque la plus importante de la télévision sur la vie démocratique. Comme l'expliquent Swanson et Mancini :

In general, the format of television favours for formal and structural reasons. Formally the medium favours the representation of human figures over complex institutions such as political parties, while structurally, the medium's commercial logic favours offering access to all candidates who can pay the cost of advertising, passing over the parties ${ }^{3}$.

En d'autres termes, la médiatisation a eu comme effet majeur de mettre les candidats individuels en avant aux dépens de leur parti. Dans le cas belge, l'exemple le plus frappant est celui des cinq teletubbies du SP.A. Freya Vandenbossche, Johan Vande Lanotte, Frank Vandenbroucke, Steve Stevaert et Patrick Janssens étaient omniprésents dans les médias flamands à l'occasion des campagnes électorales fédérales de 2003 et régionales de 2004. Ces cinq personnalités populaires prenaient clairement l'ascendant sur leur parti. Il ne s'agit toutefois pas d'un cas exceptionnel mais plutôt d'un parmi d'autres. Ainsi, aux PaysBas, le succès de la liste Pim Fortuyn en 2002 a-t-il été construit sur la popularité et la forte présence médiatique de Pim Fortuyn.

Un des corollaires de la personnalisation de la politique induite par les médias, et en particulier par la télévision, est l'émergence d'un nouveau type de candidats, les celebrity politicians ${ }^{4}$. Ceux-ci sont de

Martin Press, New York, 1992, p. 221.

1 Voir par exemple Thoveron, Gabriel, La marchandisation de la politique, Bruxelles, Labor, 2003, pp. 35-40,

2 Monière, Denis, Démocratie médiatique et représentation politique, Montréal, Presses de l'Université de Montréal, 1999, p. 11.

3 Swanson, David L. and Mancini, Paolo (eds.), op. cit., p. 13.

4 Street, John, « Celebrity Politicians: Popular Culture and Political Representation », 
deux types. D'un côté se trouvent les élus qui avaient acquis une notoriété en dehors de la sphère politique et qui s'en sont servis pour entrer avec succès dans la course électorale. Les exemples sont multiples : Ronald Reagan, Sebastian Coe (champion olympique d'athlétisme devenu député britannique), Bernard Tapie, Arnold Schwarzenegger... Le phénomène n'a d'ailleurs pas épargné la Belgique. En Flandre, les Bekende Vlamingen (BVs) sont nombreux : Jean-Marie Dedecker (VLD, entraîneur de l'équipe national de judo), Dirck Sterckx (VLD, journaliste), Anke Van Dermeersch (Vlaams Belang, ex-miss Belgique), Herman Schueremans (VLD, organisateur Rock Werchter), Chokri Mahassine (SP.A, organisateur Pukkelpop). Dans l'espace francophone également, le phénomène a pris de l'ampleur avec Marc Wilmots (MR, footballeur), Frédérique Ries (MR, journaliste), Carlo Di Antonio $(\mathrm{CDH}$, organisateur Dour Festival), Jean-Marc Nollet (Ecolo, ancien président de la FEF).

L'autre catégorie de celebrity politicians regroupe les responsables politiques qui deviennent célèbres pour leur participation à des évènements et activités qui ne sont pas directement liés à leurs fonctions politiques. Il s'agit, par exemple, d'élus qui ont pu gagner en popularité grâce à leur participation à des émissions télévisées n'ayant pas une visée politique. La présence de responsables politiques flamands à des émissions de divertissement comme les Slimste Mens a pu permettre l'émergence de cet autre type de celebrity politicians. Dans la même logique, on peut citer la participation de l'ancien président des libéraux britanniques, Charles Kennedy, à l'émission populaire Have I Got News for You?

Même si le lien célébrité-carrière politique est différent pour les deux types de celebrity politicians, la logique qui préside à leur présence accrue en politique est la même. Etant donné la centralité des médias, et surtout de la télévision, dans l'accès à l'information politique, l'aura médiatique est une ressource de plus en plus recherchée par les partis pour gagner une élection. Le fait d'être connu et présent dans les médias est une nouvelle ressource que l'aspirant aux fonctions électives peut faire valoir de la même manière qu'un éventuel ancrage local ou associatif. La télévision endosse ainsi une fonction de sélection des élites politiques ${ }^{1}$, rompant avec le parcours classique et graduel du militant qui, à force de travail et d'engagement, gravit les échelons de la noto-

British Journal of Politcs and International Relations, vol. 6, 2004, pp. 435-452.

1 Gerstlé, Jacques, La communication politique, Paris, PUF, 1993, p. 40. 
riété partisane, du local vers le national. Le militantisme, et le travail dit « de terrain », qualités nécessaires et suffisantes des leaders politiques de jadis doivent désormais se doubler de télégénie pour permettre une accession à des postes à haute responsabilité -donc à haute visibilité.

Ce processus de personnalisation de la vie politique trouve à s'exprimer institutionnellement dans certaines réformes appliquées à la vie interne des partis et à la sélection des candidats aux postes électifs. En effet, les trois partis traditionnels francophones élisent désormais leur président au suffrage universel de leurs membres, libéraux et socialistes ayant rejoint, successivement à la fin des années 1980 et à la fin des années 1990, un mouvement lancé, dès les années 1960, par le parti social chrétien. Quel moment plus évident de ratification symbolique de l'emprise d'une personne - le parti, c'est moi $^{1} \ldots$ - sur une organisation partisane que ces scrutins - souvent - courus d'avance, bien que nimbés d'une phraséologie participative ? À une échelle plus large, puisque atteignant directement l'ensemble du système partisan via des révisions du droit électoral, la diminution de moitié de l'effet dévolutif de la case de tête décidée sous le gouvernement arc-en-ciel, et, en Wallonie, la récente modification de la loi communale instaurant un semblant d'élection directe du bourgmestre participent de la même évolution vers une individualisation de la compétition politique. Ces concrétisations institutionnelles de la loi d'airain de la personnalisation ont, signalons-le, été accueillies favorablement par la plupart des opinion leaders, médiatiques ou politiques, du paysage francophone ${ }^{2}$.

À la lecture des pages précédentes, on s'aperçoit bien de l'importance des médias dans la vie politique contemporaine, mais aussi de leurs multiples influences sur le système démocratique. Dans ce contexte, une étude de la spectacularisation des personnalités politiques dans les programmes de télévision en Communauté française est plus que pertinente pour mieux cerner les enjeux multiples du lien complexe et ambigu entre médias et politiques.

En particulier, une des questions centrales qui se posent est de savoir qui domine la relation entre les médias et la politique. Pour

1 Voir Fiers, Stefaan, Partijvoorzitters in België, of 'Le parti c'est moi?', thèse de doctorat, Katholieke Universiteit Leuven, 1998.

2 Notons toutefois que socialistes et écologistes manifestèrent quelques réticences à supprimer complétement l'effet des votes en tête de liste, ce qui a conduit au compromis actuel. Voir Delwit, Pascal, Composition, décomposition et recomposition du paysage politique en Belgique, Bruxelles, Labor, 2003, p. 136. 
certains, on se trouve dans une logique médiatique où ceux-ci fixent l'agenda politique. Le choix des thèmes centraux de la vie politique et des personnalités qui font l'actualité est déterminé par la presse en fonction de ses impératifs propres ${ }^{1}$. Le monde politique se trouverait ainsi démuni, contraint de constamment humer l'air médiatique pour réagir adéquatement. C'est ainsi, nous avons déjà eu l'occasion de le souligner, que beaucoup d'analystes désenchantés s'interrogent benoîtement sur ce que font les médias à notre politique ${ }^{2}$, avec comme postulat permanent que les logiques médiatiques corrompent l'esprit public, et, partant, imposent les thèmes qui font l'actualité, thèmes sur lesquels les politiciens sont priés de se positionner. On a déjà abondamment glosé sur la prétendue mise en avant de la thématique de l'insécurité par les journaux télévisés français dans les mois précédant la présidentielle de 2002. Un des principaux bénéficiaires d'une campagne ainsi orientée a d'ailleurs admis avoir délibérément appuyé sur la question sécuritaire... à cause des multiples reportages télévisés à ce sujet. "Vous savez, je regarde aussi les journaux télévisés. Qu'est-ce que je vois depuis des mois et des mois: tous les jours, ces actes de violence, de délinquance, de criminalité. C'est bien le reflet d'une certaine situation. Ce n'est pas moi qui choisissais vos sujets » déclarait en effet Jacques Chirac à Olivier Mazerolles le 24 avril 2002³. Les médias, sans directement faire l'opinion, n'en donneraient donc pas moins le ton de l'actualité et des débats politiques. « [The media] may not be successful much of the time in telling people what to think, but it is stunningly successful in telling its readers what to think about ${ }^{4}$.

À l'inverse, d'autres auteurs considèrent que malgré leur centralité, les médias demeurent soumis aux politiques. Ce sont ces derniers qui fixent l'agenda et qui décident de qui sera présent dans la presse. Les impératifs qui déterminent la présence médiatique sont ceux des partis et non ceux de médias trop dépendants financièrement et politiquement pour pouvoir s'opposer efficacement au pouvoir des communicants ${ }^{5}$. Ces discours antagonistes sur les pouvoirs médiatiques, et en particulier de

1 Altheide, D. and Snow, R., Media Logic, Sage, Beverly Hills, 1979.

2 Voir, pour une contribution récente à cette problématique, Lloyd, John, What the Media are Doing to Our Politics, Londres, Constable, 2004.

3 Cité par Halimi, Serge, Les nouveaux chiens de garde, Paris, Raison d'agir, 2005.

4 Cohen, Bernard, The Pressa and Foreign Policy, Princeton, Princeton University Press, 1963, p. 13.

5 Voir Nobre-Correia, José-Manuel, « Journalisme: une certaine mort annoncée », in: Politique, $\mathrm{n}^{\circ}$ 37, décembre 2004, pp. 46-49. 
l'agenda-setting, recoupent la distinction sociologique entre populisme et misérabilisme, selon que l'observateur se solidarise de la condition journalistique ou de la condition politique contemporaines. Si la réalité est sans doute plus mitigée et plus complexe, il n'en reste pas moins que la question du rapport de forces entre médias et politiques est une des problématiques centrales à examiner si l'on souhaite éviter toute dérive. Un pan non négligeable de la sociologie de la communication, depuis Mc Combs et Shaw ${ }^{1}$, et en particulier dans le monde anglo-saxon, a tenté de mesurer empiriquement cette fonction d'agenda-setting, avec une réussite variable, mais qui rend indiscutables certaines observations. Le travail de synthèse mené, en Belgique, par Stefaan Walgrave, Michiel Nuytemans et Lieven De Winter est à ce titre appelé à faire rapidement figure de référence. Ces auteurs concluent notamment leurs analyses par la -modeste- assertion que les médias déterminent l'agenda politique de manière très contingente, fonction de circonstances sur lesquelles les pouvoirs médiatiques n'ont aucune prise: the where, what, who and when of political agenda-setting. Par ailleurs, cette fonction de mise à l'agenda ne prendrait toute sa mesure que sur la facette symbolique de l'agenda politique (l'ordre du jour du parlement), et moins sur sa facette institutionnelle (le programme gouvernemental) ${ }^{2}$. En tout état de cause, la perception de l'influence de la télévision s'alimente des constats plus ou moins polémiques de circulation circulaire de l'information $^{3}$ et d'uniformisation des contenus médiatiques, et en particulier de l'information télévisée. Mais les études scientifiques sur le sujet ne parviennent pas à trancher définitivement en faveur d'une homogénéité ou d'une hétérogénéité croissante des contenus informatifs télévisuels. Ainsi Sinardet, De Swert et Dandoy ont, pour la Belgique, conclu que les journaux des quatre grandes chaînes généralistes belges, publiques et privées, francophones et flamandes, gardaient des positionnement éditoriaux distincts tout au long des années nonante, la RTBF surtout présentant des contenus sensiblement différents de ceux de ses concur-

1 McCombs, Maxwell, Shaw, Donald, «The Agenda-Setting Function of Mass Media », in: The Public Opinion Quarterly, vol. 36, n² 2, été 1972, pp. 176-187.

2 De Winter, Lieven, Nuytemans, Michiel, Walgrave, Stefaan, « Specifying the media’s political agenda-setting power. Media, civil society, parliament and government in a small consociational democracy (Belgium, 1991-2000) », ECPR working paper, Uppsala, avril 2004, p. 40.

3 Bourdieu, Pierre, Sur la télévision. Suivi de l'emprise du journalisme, Paris, Raison d'Agir, 1996, p. 22. 
rentes ${ }^{1}$. La grosse moitié de sujets communs que proposent chaque jour, les deux journaux télévisés francophones ne manque toutefois pas d'alimenter les philippiques dénonçant l'empire des médias sur la société (voir la suite de ce rapport), lancés parfois des bancs politiques. De fait l'observateur engagé y verra, selon le sens de son engagement, misérabiliste ou populiste, un verre à moitié vide ou à moitié plein.

1 Dandoy, Régis, De Swert, Knut, Sinardet, Dave, «Les sujets des journaux télévisés francophones et flamands. Une comparaison longitudinale », in: Courrier Hebdomadaire du CRISP, $\mathrm{n}^{\circ}$ 1864, 2004. 
